\title{
First-order nonlinear differential equations with state-dependent impulses
}

\author{
Lukáš Rachůnek and Irena Rachůnková*
}

\author{
"Correspondence: \\ irena.rachunkova@upol.cz \\ Department of Mathematics, \\ Faculty of Science, Palacký \\ University, 17. listopadu 12, \\ Olomouc, 77146, Czech Republic
}

\begin{abstract}
The paper deals with the state-dependent impulsive problem

$$
\begin{aligned}
& z^{\prime}(t)=f(t, z(t)) \quad \text { for a.e. } t \in[a, b] \\
& z(\tau+)-z(\tau)=\mathcal{J}(\tau, z(\tau)), \quad \gamma(z) \\
& \ell(z)=c_{0}
\end{aligned}
$$$$
z(\tau+)-z(\tau)=\mathcal{J}(\tau, z(\tau)), \quad \gamma(z(\tau))=\tau,
$$

where $[a, b] \subset \mathbb{R}, c_{0} \in \mathbb{R}, f$ fulfils the Carathéodory conditions on $[a, b] \times \mathbb{R}$, the impulse function $\mathcal{J}$ is continuous on $[a, b] \times \mathbb{R}$, the barrier function $\gamma$ has a continuous first derivative on some subset of $\mathbb{R}$ and $\ell$ is a linear bounded functional which is defined on the Banach space of left-continuous regulated functions on $[a, b]$ equipped with the sup-norm. The functional $\ell$ is represented by means of the Kurzweil-Stieltjes integral and covers all linear boundary conditions for solutions of first-order differential equations subject to state-dependent impulse conditions. Here, sufficient and effective conditions guaranteeing the solvability of the above problem are presented for the first time.
\end{abstract}

MSC: 34B37; 34B15

Keywords: first-order ODE; state-dependent impulses; transversality conditions; general linear boundary conditions; existence; Kurzweil-Stieltjes integral

\section{Introduction}

The investigation of impulsive differential equations has a long history; see, e.g., the monographs [1-3]. Most papers dealing with impulsive differential equations subject to boundary conditions focus their attention on impulses at fixed moments. But this is a very particular case of a more complicated case with state-dependent impulses. Boundary value problems with state-dependent impulses, where difficulties with an operator representation appear ( $c f$. Remark 6.2), are substantially less developed. We refer to the papers [4-6] and [7] which are devoted to periodic problems, and for problems with other boundary conditions, see $[8,9]$ or $[10-12]$.

Here, in our paper, we present an approach leading to a new existence principle for impulsive boundary value problems. This approach is applicable to each linear boundary condition which is considered with some first-order differential equation subject to statedependent impulses. The important step is a proof of a transversality (Remark 2.3 and Lemmas 5.1 and 5.2), which makes possible a construction of a continuous operator (Section 6) whose fixed point leads to a solution of our original impulsive problem (Section 7). 


\section{Notation}

Let $M \subset \mathbb{R}^{n}, n \in \mathbb{N},[a, b] \subset \mathbb{R}$.

- $\mathbb{C}(M)$ is the set of real functions continuous on $M$.

- $\mathbb{A} \mathbb{C}(M)$ is the set of real functions absolutely continuous on $M$.

- $\mathbb{L}^{1}[a, b]$ is the set of real functions Lebesgue integrable on $[a, b]$.

- $\mathbb{L}^{\infty}[a, b]$ is the set of real functions essentially bounded on $[a, b]$.

- $\mathbb{B V}[a, b]$ is the set of real functions with bounded variation on $[a, b]$.

- $\mathbb{G}_{L}[a, b]$ is the set of real left-continuous regulated functions on $[a, b]$, that is, $z \in \mathbb{G}_{L}[a, b]$ if and only if $z:[a, b] \rightarrow \mathbb{R}$, and for each $\tau_{1} \in(a, b]$ and each $\tau_{2} \in[a, b)$,

$$
z\left(\tau_{1}\right)=z\left(\tau_{1}-\right)=\lim _{t \rightarrow \tau_{1^{-}}} z(t), \quad z\left(\tau_{2}+\right)=\lim _{t \rightarrow \tau_{2}+} z(t) \in \mathbb{R} .
$$

- $\operatorname{Car}([a, b] \times M)$ is the set of functions $f:[a, b] \times M \rightarrow \mathbb{R}$ such that

(i) $f(\cdot, x):[a, b] \rightarrow \mathbb{R}$ is measurable for all $x \in M$,

(ii) $f(t, \cdot): M \rightarrow \mathbb{R}$ is continuous for a.e. $t \in[a, b]$,

(iii) for each compact set $Q \subset M$, there exists $m_{Q} \in \mathbb{L}^{1}[a, b]$ satisfying

$$
|f(t, x)| \leq m_{Q}(t) \quad \text { for a.e. } t \in[a, b] \text { and each } x \in Q \text {. }
$$

- The set $\mathbb{L}^{\infty}[a, b]$ equipped with the norm

$$
\|z\|_{\infty}=\operatorname{supess}\{|z(t)|: t \in[a, b]\} \quad \text { for } z \in \mathbb{L}^{\infty}[a, b]
$$

is a Banach space.

- Since $\mathbb{C}[a, b] \subset \mathbb{G}_{L}[a, b] \subset \mathbb{L}^{\infty}[a, b]$, we equip the sets $\mathbb{C}[a, b]$ and $\mathbb{G}_{L}[a, b]$ with the norm $\|\cdot\|_{\infty}$ and get also Banach spaces (cf. [13]). Then (1.2) can be written as

$$
\|z\|_{\infty}=\sup \{|z(t)|: t \in[a, b]\} \quad \text { for } z \in \mathbb{G}_{L}[a, b]
$$

and

$$
\|z\|_{\infty}=\max \{|z(t)|: t \in[a, b]\} \quad \text { for } z \in \mathbb{C}[a, b] .
$$

- $\mathbb{W}^{1, \infty}[a, b]$ is the Banach space of functions $z:[a, b] \rightarrow \mathbb{R}$ such that $z \in \mathbb{A} \mathbb{C}[a, b]$ and $z^{\prime} \in \mathbb{L}^{\infty}[a, b]$, where the norm $\|\cdot\|_{1, \infty}$ is given by

$$
\|z\|_{1, \infty}=\|z\|_{\infty}+\left\|z^{\prime}\right\|_{\infty} \quad \text { for } z \in \mathbb{W}^{1, \infty}[a, b] .
$$

- $\chi_{A}$ is the characteristic function of a set $A$, where $A \subset \mathbb{R}$.

\section{Formulation of problem}

We investigate the solvability of the nonlinear differential equation

$$
z^{\prime}(t)=f(t, z(t))
$$

subject to the state-dependent impulse condition

$$
z(\tau+)-z(\tau)=\mathcal{J}(\tau, z(\tau)), \quad \gamma(z(\tau))=\tau,
$$


and the general linear boundary condition

$$
\ell(z)=c_{0}
$$

Here we assume that

$$
\left\{\begin{array}{l}
f \in \operatorname{Car}([a, b] \times \mathbb{R}), \quad \mathcal{J} \in \mathbb{C}([a, b] \times \mathbb{R}), \quad[a, b] \subset \mathbb{R}, \\
K \in(0, \infty), \quad \gamma \in \mathbb{C}^{1}[-K, K], \quad c_{0} \in \mathbb{R},
\end{array}\right.
$$

and $\ell: \mathbb{G}_{L}[a, b] \rightarrow \mathbb{R}$ is a linear bounded functional.

Definition 2.1 A function $z:[a, b] \rightarrow \mathbb{R}$ is a solution of problem (2.1), (2.2) if

- there exists a unique $\tau \in(a, b)$ such that $\gamma(z(\tau))=\tau$;

- the restrictions $\left.z\right|_{[a, \tau]}$ and $\left.z\right|_{(\tau, b]}$ are absolutely continuous;

- $z(\tau+)=z(\tau)+\mathcal{J}(\tau, z(\tau))$

- $z$ satisfies equation (2.1) for a.e. $t \in[a, b]$.

Definition 2.2 A graph of a function $\gamma:[-K, K] \rightarrow \mathbb{R}$ is called a barrier $\gamma$.

Remark 2.3 Let $\mathcal{S}$ be the set of all solutions of problem (2.1), (2.2). According to Definition 2.1, each function $z \in \mathcal{S}$ satisfies a transversality property, which means that the graph of $z$ crosses a barrier $\gamma$ at a unique point $\tau \in(a, b)$, where the impulse $\mathcal{J}$ acts on $z$. After that (for $t \in(\tau, b]$ ) the graph of $z$ lies on the right of the barrier $\gamma$. This transversality property follows from transversality conditions (cf. (4.5), (4.6)) and it is proved in Section 5.

Assume that $z_{1}, z_{2} \in \mathcal{S}$ and $z_{1} \neq z_{2}$. Then there exists a unique $\tau_{i} \in(a, b)$ such that $\gamma\left(z_{i}\left(\tau_{i}\right)\right)=\tau_{i}$ for $i=1,2$ and $\tau_{1} \neq \tau_{2}$ can occur. Therefore different functions from $\mathcal{S}$ can have their discontinuities at different points from $(a, b)$. Our aim in this paper is to prove the existence of a solution of problem (2.1), (2.2) satisfying the general linear boundary condition (2.3). To do this, we need a suitable linear space containing $\mathcal{S}$. Due to state-dependent impulses, the Banach space of piece-wise continuous functions on $[a, b]$ with the sup-norm cannot be used here. Therefore we choose the Banach space $\mathbb{G}_{L}[a, b]$. Clearly, by (1.1), $\mathcal{S} \subset \mathbb{G}_{L}[a, b]$. The operator $\ell$ in the general linear boundary condition (2.3) can be written uniquely in the form

$$
\ell(z)=k z(a)+{ }_{(\mathrm{KS})} \int_{a}^{b} v(t) \mathrm{d}[z(t)]
$$

where $k \in \mathbb{R}, v \in \mathbb{B V}[a, b]$ and ${ }_{(\mathrm{KS})} \int_{a}^{b}$ is the Kurzweil-Stieltjes integral (cf. [14], Theorem 3.8). Representation (2.5) is correct on $\mathcal{S}$, because for each $z \in \mathbb{G}_{L}[a, b]$ the integral (KS) $\int_{a}^{b} v(t) \mathrm{d}[z(t)]$ exists. Its definition and properties can be found in [15] (see PerronStieltjes integral based on the work of Kurzweil).

Definition 2.4 A function $z:[a, b] \rightarrow \mathbb{R}$ is a solution of problem (2.1)-(2.3) if $z$ is a solution of problem (2.1), (2.2) and fulfils (2.3). 


\section{Green's function}

For further investigation, we will need a linear homogeneous problem corresponding to problem (2.1)-(2.3). Such problem has the form

$$
\begin{aligned}
& z^{\prime}(t)=0, \\
& \ell(z)=0,
\end{aligned}
$$

because the impulse in (2.2) disappears if $\mathcal{J} \equiv 0$. We will also work with the nonhomogeneous equation

$$
z^{\prime}(t)=q(t)
$$

where $q \in \mathbb{L}^{1}[a, b]$.

Definition 3.1 A solution of problem (3.3), (3.2) is a function $z \in \mathbb{A}[a, b]$ satisfying equation (3.3) for a.e. $t \in[a, b]$ and fulfilling condition (3.2).

Remark 3.2 If $x$ is a solution of problem (3.3), (3.2), then $x$ belongs to $\mathbb{A} \mathbb{C}[a, b]$, and consequently condition (3.2) can be written in the form (cf. (2.5))

$$
\ell(x)=k x(a)+\int_{a}^{b} v(t) x^{\prime}(t) \mathrm{d} t=0
$$

where $k \in \mathbb{R}, v \in \mathbb{B} \mathbb{V}$ and the Lebesgue integral $\int_{a}^{b} v(t) x^{\prime}(t) \mathrm{d} t$ is used.

Definition 3.3 A function $G:[a, b] \times[a, b] \rightarrow \mathbb{R}$ is the Green's function of problem (3.1), (3.2) if

(i) for any $s \in(a, b)$, the restrictions $\left.G(\cdot, s)\right|_{[a, s)},\left.G(\cdot, s)\right|_{(s, b]}$ are solutions of equation (3.1) and $G(s+, s)-G(s, s)=1$, where $G(s, s)=G(s-, s)$;

(ii) $G(t, \cdot) \in \mathbb{B V}[a, b]$ for any $t \in[a, b]$;

(iii) for any $q \in \mathbb{L}^{1}[a, b]$, the function

$$
x(t)=\int_{a}^{b} G(t, s) q(s) \mathrm{d} s
$$

fulfils condition (3.4).

Lemma 3.4 Let $\ell$ be from (2.5) with $k \in \mathbb{R}$ and $v \in \mathbb{B V}[a, b]$.

(i) $k \neq 0$ if and only if there exists the Green's function $G$ of problem (3.1), (3.2) which has the form

$$
G(t, s)= \begin{cases}-\frac{\nu(s)}{k} & \text { for } a \leq t \leq s \leq b \\ 1-\frac{\nu(s)}{k} & \text { for } a \leq s<t \leq b\end{cases}
$$

(ii) $k \neq 0$ if and only if there exists a unique solution $x$ of problem (3.3), (3.4), which has a form of (3.5) with G from (3.6). 
Proof Clearly, $G$ given by (3.6) fulfils (i) and (ii) of Definition 3.3 if and only if $k \neq 0$. A general solution of equation (3.3) is $x(t)=c+\int_{a}^{t} q(s) \mathrm{d} s$, where $c \in \mathbb{R}$. By (3.4),

$$
\ell(x)=k c+\int_{a}^{b} v(t) q(t) \mathrm{d} t=0
$$

The equation

$$
k c=-\int_{a}^{b} v(t) q(t) \mathrm{d} t
$$

has a unique solution $c$ if and only if $k \neq 0$. Then a unique solution $x$ of problem (3.3), (3.4) is written as

$$
\begin{aligned}
x(t) & =-\frac{1}{k} \int_{a}^{b} v(s) q(s) \mathrm{d} s+\int_{a}^{t} q(s) \mathrm{d} s \\
& =\int_{a}^{t}\left(1-\frac{v(s)}{k}\right) q(s) \mathrm{d} s+\int_{t}^{b}\left(-\frac{v(s)}{k}\right) q(s) \mathrm{d} s, \quad t \in[a, b] .
\end{aligned}
$$

Lemma 3.5 Let $G$ be the Green's function of problem (3.1), (3.2), where $\ell$ is from (2.5) and $k \neq 0$. Then, for each $s \in[a, b)$, the function $G(\cdot, s)$ belongs to $\mathbb{G}_{L}[a, b]$ and

$$
\ell(G(\cdot, s))=0, \quad s \in[a, b) .
$$

Proof Choose $s \in[a, b)$. By (3.6),

$$
G(t, s)=\chi_{(s, b]}(t)-\frac{v(s)}{k} \quad \text { for } t \in[a, b] .
$$

Consequently, the function $G(\cdot, s)$ belongs to $\mathbb{G}_{L}[a, b]$. This yields that the integral (KS) $\int_{a}^{b} v(t) \mathrm{d}[G(t, s)]$ exists for each $v \in \mathbb{B V}[a, b]$. Note that since $G(\cdot, s)$ is not continuous on $[a, b]$, formula (3.4) cannot be used for $G(\cdot, s)$ in place of $x$. Instead, we use the properties of the Kurzweil-Stieltjes integral which justify the following computation

$$
\begin{aligned}
(\mathrm{KS}) \int_{a}^{b} v(t) \mathrm{d}[G(t, s)] & =(\mathrm{KS}) \int_{a}^{b} v(t) \mathrm{d}\left[\chi_{(s, b]}(t)-\frac{v(s)}{k}\right] \\
& =(\mathrm{KS}) \int_{a}^{b} v(t) \mathrm{d}\left[\chi_{(s, b]}(t)\right]-(\mathrm{KS}) \int_{a}^{b} v(t) \mathrm{d}\left[\frac{v(s)}{k}\right]=v(s) .
\end{aligned}
$$

Hence, by (2.5), we get

$$
\ell(G(\cdot, s))=k G(a, s)+(K S) \int_{a}^{b} v(t) \mathrm{d}[G(t, s)]=k\left(\frac{-v(s)}{k}\right)+v(s)=0 .
$$

Example 3.6 Consider a solution $x$ of problem (3.3), (3.2), where $\ell$ has a form of the twopoint boundary condition

$$
\ell(x)=\alpha x(a)+\beta x(b)=0, \quad \alpha, \beta \in \mathbb{R} .
$$


We will show that $\ell$ can be expressed in a form of (3.4). If $\alpha+\beta \neq 0$, then $k$ and $v$ can be found from the equality

$$
\alpha x(a)+\beta x(b)=k x(a)+\int_{a}^{b} v(t) x^{\prime}(t) \mathrm{d} t .
$$

Assuming that $v(t) \equiv v_{0} \in \mathbb{R}$, we get

$$
\alpha x(a)+\beta x(b)=k x(a)+v_{0}(x(b)-x(a)),
$$

and hence $k=\alpha+\beta, v_{0}=\beta$. In addition, if $\alpha+\beta \neq 0$, then (cf. (3.6))

$$
G(t, s)= \begin{cases}-\frac{\beta}{\alpha+\beta} & \text { for } a \leq t \leq s \leq b, \\ 1-\frac{\beta}{\alpha+\beta} & \text { for } a \leq s<t \leq b .\end{cases}
$$

Example 3.7 Consider a solution $x$ of problem (3.3), (3.2), where $\ell$ has a form of the multipoint boundary condition

$$
\ell(x)=\sum_{i=0}^{n} \alpha_{i} x\left(t_{i}\right), \quad \alpha_{i} \in \mathbb{R}, i=0,1, \ldots, n, n \in \mathbb{N} .
$$

Here $a=t_{0}<t_{1}<\cdots<t_{n}=b$. If $\sum_{i=0}^{n} \alpha_{i} \neq 0$, then $k$ and $v$ of (3.4) can be found from the equality

$$
\sum_{i=0}^{n} \alpha_{i} x\left(t_{i}\right)=k x(a)+\int_{a}^{b} v(t) x^{\prime}(t) \mathrm{d} t
$$

Assume that $v$ is a piece-wise constant right-continuous function on $[a, b]$, that is,

$$
\begin{aligned}
& v(s)=v_{i} \quad \text { for } s \in\left[t_{i}, t_{i+1}\right), i=0, \ldots, n-2, \\
& v(s)=v_{n-1} \quad \text { for } s \in\left[t_{n-1}, b\right],
\end{aligned}
$$

where $v_{i} \in \mathbb{R}, i=0, \ldots, n-1$. By (3.10), we get

$$
\begin{aligned}
\sum_{i=0}^{n} \alpha_{i} x\left(t_{i}\right) & =k x(a)+\sum_{i=0}^{n-1} v_{i} \int_{t_{i}}^{t_{i+1}} x^{\prime}(t) \mathrm{d} t \\
& =k x(a)+v_{0}\left(x\left(t_{1}\right)-x(a)\right)+v_{1}\left(x\left(t_{2}\right)-x\left(t_{1}\right)\right)+\cdots+v_{n-1}\left(x(b)-x\left(t_{n-1}\right)\right) .
\end{aligned}
$$

Consequently,

$$
v_{i}=\sum_{j=i+1}^{n} \alpha_{j}, \quad i=0, \ldots, n-1, \quad k=\sum_{j=0}^{n} \alpha_{j} .
$$

To summarize, if $\sum_{j=0}^{n} \alpha_{j} \neq 0$, then

$$
\begin{aligned}
& v(s)=\sum_{j=i+1}^{n} \alpha_{j} \quad \text { for } s \in\left[t_{i}, t_{i+1}\right), i=0, \ldots, n-2, \\
& v(s)=\alpha_{n} \quad \text { for } s \in\left[t_{n-1}, b\right],
\end{aligned}
$$


and further (cf. (3.6))

$$
G(t, s)= \begin{cases}-\frac{v(s)}{\sum_{j=0}^{n} \alpha_{j}} & \text { for } a \leq t \leq s \leq b, \\ 1-\frac{\nu(s)}{\sum_{j=0}^{n} \alpha_{j}} & \text { for } a \leq s<t \leq b .\end{cases}
$$

Example 3.8 Consider a solution $x$ of problem (3.3), (3.2), where $\ell$ has a form of the integral condition

$$
\ell(x)=x(b)-\int_{a}^{b} h(\xi) x(\xi) \mathrm{d} \xi
$$

where $h \in \mathbb{L}^{1}[a, b]$. If $\int_{a}^{b} h(\xi) \mathrm{d} \xi \neq 1$, then $k$ and $v$ of (3.4) can be found from the equality

$$
x(b)-\int_{a}^{b} h(\xi) x(\xi) \mathrm{d} \xi=k x(a)+\int_{a}^{b} v(t) x^{\prime}(t) \mathrm{d} t .
$$

Let us put

$$
v(s)=\int_{a}^{s} h(\xi) \mathrm{d} \xi+v(a)
$$

Then

$$
\int_{a}^{b} v(\xi) x^{\prime}(\xi) \mathrm{d} \xi=-\int_{a}^{b} h(\xi) x(\xi) \mathrm{d} \xi+v(b) x(b)-v(a) x(a)
$$

and (3.11) gives $v(a)=k, \int_{a}^{b} h(\xi) \mathrm{d} \xi+k=1$. Consequently,

$$
k=1-\int_{a}^{b} h(\xi) \mathrm{d} \xi, \quad v(s)=1-\int_{s}^{b} h(\xi) \mathrm{d} \xi, \quad s \in[a, b] .
$$

Similarly, if

$$
\ell(x)=x(a)-\int_{a}^{b} h(\xi) x(\xi) \mathrm{d} \xi
$$

and $\int_{a}^{b} h(\xi) \mathrm{d} \xi \neq 1$, we derive

$$
k=1-\int_{a}^{b} h(\xi) \mathrm{d} \xi, \quad v(s)=-\int_{s}^{b} h(\xi) \mathrm{d} \xi, \quad s \in[a, b] .
$$

In both cases, $G$ is written as

$$
G(t, s)= \begin{cases}-\frac{v(s)}{1-\int_{a}^{b} h(\xi) \mathrm{d} \xi} & \text { for } a \leq t \leq s \leq b, \\ 1-\frac{\nu(s)}{1-\int_{a}^{b} h(\xi) \mathrm{d} \xi} & \text { for } a \leq s<t \leq b .\end{cases}
$$




\section{Assumptions}

An existence result for problem (2.1)-(2.3) will be proved in the next sections under the basic assumption (2.4) and the following additional assumptions imposed on $f, \ell, \mathcal{J}$ and $\gamma$.

(i) Boundedness of $f$

$$
\left\{\begin{array}{l}
\text { There exists } h \in \mathbb{L}^{\infty}[a, b] \text { such that } \\
|f(t, x)| \leq h(t) \text { for a.e. } t \in[a, b] \text { and all } x \in \mathbb{R} .
\end{array}\right.
$$

(ii) Boundedness of $\mathcal{J}$

$$
\left\{\begin{array}{l}
\text { There exists } J_{0} \in(0, \infty) \text { such that } \\
|\mathcal{J}(t, x)| \leq J_{0} \quad \text { for } t \in[a, b], x \in \mathbb{R} .
\end{array}\right.
$$

(iii) Boundedness of $\gamma$

$$
\left\{\begin{array}{l}
\text { There exist } a_{1}, b_{1} \in(a, b) \text { such that } \\
a_{1} \leq \gamma(x) \leq b_{1} \quad \text { for } x \in[-K, K]
\end{array}\right.
$$

(iv) Properties of $\ell$

$$
\ell \text { fulfils (2.5), where } k \in \mathbb{R}, k \neq 0, v \in \mathbb{B V}[a, b] \cap \mathbb{C}\left[a_{1}, b_{1}\right] \text {. }
$$

(v) Transversality conditions

$$
\begin{aligned}
& \left|\gamma^{\prime}(x)\right|<\frac{1}{\|h\|_{\infty}} \quad \text { for } x \in[-K, K], \\
& \left\{\begin{array}{llll}
\text { either } & \mathcal{J}(t, x) \geq 0, & \gamma^{\prime}(x) \leq 0 & \text { for } t \in\left[a_{1}, b_{1}\right], x \in[-K, K], \\
\text { or } & \mathcal{J}(t, x) \leq 0, & \gamma^{\prime}(x) \geq 0 & \text { for } t \in\left[a_{1}, b_{1}\right], x \in[-K, K],
\end{array}\right.
\end{aligned}
$$

where $h$ is from (4.1) and $a_{1}, b_{1}$ are from (4.3).

(vi) $\mathbb{L}^{\infty}$-continuity of $f$

$$
\left\{\begin{array}{l}
\text { For any } \varepsilon>0, \text { there exists } \delta>0 \text { such that } \\
|x-y|<\delta \Rightarrow\|f(\cdot, x)-f(\cdot, y)\|_{\infty}<\varepsilon, \quad x, y \in[-K, K] .
\end{array}\right.
$$

\section{Remark 4.1}

(a) Boundedness of $f$ and $\mathcal{J}$ can be replaced by more general conditions, for example, growth or sign ones, if the method of a priori estimates is used. See, e.g., [16, 17].

(b) Continuity of $v$ on $\left[a_{1}, b_{1}\right]$ is necessary for the construction of a continuous operator in Section 6. Note that then we need $t_{1}, \ldots, t_{n-1} \notin\left[a_{1}, b_{1}\right]$ in Example 3.7.

(c) Clearly, if $f$ is continuous on $[a, b] \times[-K, K]$, then $f$ fulfils (4.7).

(d) Let there exist $p \in \mathbb{N}, \psi \in \mathbb{L}^{\infty}[a, b]$ and $g_{i} \in \mathbb{C}(\mathbb{R}), i=1, \ldots, p$, such that

$$
|f(t, x)-f(t, y)| \leq \psi(t) \sum_{i=1}^{p}\left|g_{i}(x)-g_{i}(y)\right|
$$


for a.e. $t \in[a, b]$ and all $x, y \in[-K, K]$. Then $f$ fulfils (4.7). An example of such a function $f$ is

$$
f(t, x)=\sum_{i=1}^{p} f_{i}(t) g_{i}(x)+f_{0}(t)
$$

where $f_{j} \in \mathbb{L}^{\infty}[a, b], j=0,1, \ldots, p, g_{i} \in \mathbb{C}[-K, K], i=1, \ldots, p$.

\section{Transversality}

Consider $K \in(0, \infty), h \in \mathbb{L}^{\infty}[a, b]$ and define a set $\mathcal{B}$ by

$$
\mathcal{B}=\left\{u \in \mathbb{W}^{1, \infty}[a, b]:\|u\|_{\infty}<K,\left\|u^{\prime}\right\|_{\infty}<\|h\|_{\infty}\right\} .
$$

The following two lemmas for functions from $\mathcal{B}$ are the modifications of lemmas in [10] and provide the transversality ( $c f$. Remark 2.3 ) which will be essential for operator constructions in Section 6.

Lemma 5.1 Let $\gamma$ satisfy (2.4), (4.3) and (4.5). Then, for each $u \in \overline{\mathcal{B}}$, there exists a unique $\tau \in(a, b)$ such that

$$
\tau=\gamma(u(\tau))
$$

In addition $\tau \in\left[a_{1}, b_{1}\right]$.

Proof Let us take an arbitrary $u \in \overline{\mathcal{B}}$ and denote

$$
\sigma(t)=\gamma(u(t))-t, \quad t \in[a, b] .
$$

Then, by (2.4) and (5.1), we see that $\sigma \in \mathbb{A} \mathbb{C}[a, b]$ and

$$
\sigma^{\prime}(t)=\gamma^{\prime}(u(t)) u^{\prime}(t)-1 \quad \text { for a.e. } t \in[a, b] .
$$

Since $u(a), u(b) \in[-K, K]$, condition (4.3) gives

$$
\begin{aligned}
& \sigma(a)=\gamma(u(a))-a \geq a_{1}-a>0, \\
& \sigma(b)=\gamma(u(b))-b \leq b_{1}-b<0 .
\end{aligned}
$$

Consequently, there exists at least one zero of $\sigma$ in $(a, b)$. Let $\tau \in(a, b)$ be a zero of $\sigma$. By virtue of (4.5) and (5.1), we get, for $t \in[a, b], t \neq \tau$,

$$
\begin{aligned}
\operatorname{sign}(t-\tau) \sigma(t) & =\operatorname{sign}(t-\tau) \int_{\tau}^{t} \sigma^{\prime}(s) \mathrm{d} s=\operatorname{sign}(t-\tau) \int_{\tau}^{t}\left(\gamma^{\prime}(u(s)) u^{\prime}(s)-1\right) \mathrm{d} s \\
& \leq \operatorname{sign}(t-\tau) \int_{\tau}^{t}\left(\left|\gamma^{\prime}(u(s))\right| \cdot\left\|u^{\prime}\right\|_{\infty}-1\right) \mathrm{d} s \\
& <\operatorname{sign}(t-\tau) \int_{\tau}^{t}\left(\frac{1}{\|h\|_{\infty}}\|h\|_{\infty}-1\right) \mathrm{d} s=0 .
\end{aligned}
$$


That is,

$$
\sigma>0 \quad \text { on }[a, \tau), \quad \sigma<0 \quad \text { on }(\tau, b] .
$$

Hence $\tau$ is a unique zero of $\sigma$, and (4.3) yields $\tau \in\left[a_{1}, b_{1}\right]$.

Due to Lemma 5.1, we can define a functional $\mathcal{P}: \overline{\mathcal{B}} \rightarrow\left[a_{1}, b_{1}\right]$ by

$$
\mathcal{P} u=\tau,
$$

where $\tau$ fulfils (5.2).

Lemma 5.2 Let $\gamma$ satisfy (2.4), (4.3) and (4.5). Then the functional $\mathcal{P}$ is continuous.

Proof Let us choose a sequence $\left\{u_{n}\right\}_{n=1}^{\infty} \subset \overline{\mathcal{B}}$ which is convergent in $\mathbb{W}^{1, \infty}[a, b]$. Then

$$
u_{n} \in \mathbb{W}^{1, \infty}[a, b], \quad\left\|u_{n}\right\|_{\infty} \leq K, \quad\left\|u_{n}^{\prime}\right\|_{\infty} \leq\|h\|_{\infty}, \quad n \in \mathbb{N},
$$

and there exists $u \in \mathbb{W}^{1, \infty}[a, b]$ such that

$$
\lim _{n \rightarrow \infty}\left\|u_{n}-u\right\|_{1, \infty}=0
$$

So, by virtue of (1.5) and (5.5),

$$
\begin{aligned}
& \|u\|_{\infty} \leq \lim _{n \rightarrow \infty}\left\|u-u_{n}\right\|_{\infty}+\lim _{n \rightarrow \infty}\left\|u_{n}\right\|_{\infty} \leq K, \\
& \left\|u^{\prime}\right\|_{\infty} \leq \lim _{n \rightarrow \infty}\left\|u^{\prime}-u_{n}^{\prime}\right\|_{\infty}+\lim _{n \rightarrow \infty}\left\|u_{n}^{\prime}\right\|_{\infty} \leq\|h\|_{\infty} .
\end{aligned}
$$

We see that $u \in \overline{\mathcal{B}}$. For $n \in \mathbb{N}$, define

$$
\sigma_{n}(t)=\gamma\left(u_{n}(t)\right)-t, \quad \sigma(t)=\gamma(u(t))-t, \quad t \in[a, b] .
$$

By Lemma 5.1,

$$
\sigma_{n}\left(\tau_{n}\right)=0, \quad \sigma(\tau)=0, \quad \text { where } \tau_{n}=\mathcal{P} u_{n}, \tau=\mathcal{P} u, n \in \mathbb{N} .
$$

We need to prove that

$$
\lim _{n \rightarrow \infty} \tau_{n}=\tau .
$$

Conditions (2.4), (1.5) and (5.6) yield

$$
\lim _{n \rightarrow \infty} \sigma_{n}=\sigma \quad \text { in } \mathbb{C}[a, b] .
$$

Let us take an arbitrary $\varepsilon>0$. By (5.3) and (5.9) we can find $\xi \in(\tau-\varepsilon, \tau), \eta \in(\tau, \tau+\varepsilon)$ and $n_{0} \in \mathbb{N}$ such that $\sigma_{n}(\xi)>0, \sigma_{n}(\eta)<0$ for each $n \geq n_{0}$. By Lemma 5.1 and the continuity of $\sigma_{n}$, we see that $\tau_{n} \in(\xi, \eta) \subset(\tau-\varepsilon, \tau+\varepsilon)$ for $n \geq n_{0}$, and (5.8) follows. 


\section{Fixed point problem}

In this section we assume that

conditions (2.4), (4.1)-(4.7) are fulfilled,

and we construct a fixed point problem whose solvability leads to a solution of problem (2.1)-(2.3). To this aim, having the set $\mathcal{B}$ from (5.1), we define a set $\Omega$ by

$$
\Omega=\mathcal{B} \times \mathcal{B} \subset \mathbb{W}^{1, \infty}[a, b] \times \mathbb{W}^{1, \infty}[a, b]
$$

and for $u=\left(u_{1}, u_{2}\right) \in \Omega$, we define a function $f_{u}:[a, b] \rightarrow \mathbb{R}$ as follows. We set, for a.e. $t \in[a, b]$,

$$
f_{u}(t)= \begin{cases}f\left(t, u_{1}(t)\right) & \text { if } t \in\left[a, \mathcal{P} u_{1}\right] \\ f\left(t, u_{2}(t)\right) & \text { if } t \in\left(\mathcal{P} u_{1}, b\right]\end{cases}
$$

where $\mathcal{P}$ is defined by (5.4) and the point $\mathcal{P} u_{1} \in\left[a_{1}, b_{1}\right]$ is uniquely determined due to Lemma 5.1. By (4.1)

$$
f_{u} \in \mathbb{L}^{\infty}[a, b], \quad\left\|f_{u}\right\|_{\infty} \leq\|h\|_{\infty} .
$$

Now, we can define an operator $\mathcal{F}: \bar{\Omega} \rightarrow \mathbb{W}^{1, \infty}[a, b] \times \mathbb{W}^{1, \infty}[a, b]$ by $\mathcal{F}\left(u_{1}, u_{2}\right)=\left(x_{1}, x_{2}\right)$, where

$$
\begin{aligned}
& x_{1}(t)= \begin{cases}\int_{a}^{b} G(t, s) f_{u}(s) \mathrm{d} s+\frac{c_{0}}{k} \\
\quad-\frac{v\left(\mathcal{P} u_{1}\right)}{k} \mathcal{J}\left(\mathcal{P} u_{1}, u_{1}\left(\mathcal{P} u_{1}\right)\right) & \text { if } t \leq \mathcal{P}_{u_{1}}, \\
\int_{a}^{b} G(t, s) f\left(s, u_{1}(s)\right) \mathrm{d} s+\frac{c_{0}}{k} & \\
\quad-\frac{v\left(\mathcal{P} u_{1}\right)}{k} \mathcal{J}\left(\mathcal{P} u_{1}, u_{1}\left(\mathcal{P} u_{1}\right)\right)+\mathcal{A}_{1} u & \text { if } t>\mathcal{P}_{u_{1}},\end{cases} \\
& x_{2}(t)=\left\{\begin{array}{cc}
\int_{a}^{b} G(t, s) f\left(s, u_{2}(s)\right) \mathrm{d} s+\frac{c_{0}}{k} & \\
\quad+\left(1-\frac{v\left(\mathcal{P} u_{1}\right)}{k}\right) \mathcal{J}\left(\mathcal{P} u_{1}, u_{1}\left(\mathcal{P} u_{1}\right)\right)+\mathcal{A}_{2} u & \text { if } t \leq \mathcal{P}_{u_{1}}, \\
\int_{a}^{b} G(t, s) f_{u}(s) \mathrm{d} s+\frac{c_{0}}{k} & \text { if } t>\mathcal{P}_{u_{1}} . \\
\quad+\left(1-\frac{v\left(\mathcal{P} u_{1}\right)}{k}\right) \mathcal{J}\left(\mathcal{P} u_{1}, u_{1}\left(\mathcal{P} u_{1}\right)\right) &
\end{array}\right.
\end{aligned}
$$

Here the functionals $\mathcal{A}_{1}: \bar{\Omega} \rightarrow \mathbb{R}$ and $\mathcal{A}_{2}: \bar{\Omega} \rightarrow \mathbb{R}$ are defined such that the functions $x_{1}$ and $x_{2}$ are continuous at the point $\mathcal{P} u_{1}$. Therefore

$$
\left\{\begin{array}{l}
\mathcal{A}_{1} u=\int_{a}^{b} G\left(\mathcal{P} u_{1}, s\right) f_{u}(s) \mathrm{d} s-\int_{a}^{b} G\left(\mathcal{P} u_{1}, s\right) f\left(s, u_{1}(s)\right) \mathrm{d} s \\
\mathcal{A}_{2} u=\int_{a}^{b} G\left(\mathcal{P} u_{1}, s\right) f_{u}(s) \mathrm{d} s-\int_{a}^{b} G\left(\mathcal{P} u_{1}, s\right) f\left(s, u_{2}(s)\right) \mathrm{d} s
\end{array}\right.
$$

Differentiating (6.5) and using (3.6) and (6.3), we get

$$
x_{i}^{\prime}(t)=f\left(t, u_{i}(t)\right) \quad \text { for a.e. } t \in[a, b], i=1,2 .
$$


This together with (4.1) yields

$$
\left\|x_{i}^{\prime}\right\|_{\infty} \leq\|h\|_{\infty}, \quad i=1,2
$$

Since $v \in \mathbb{B} \mathbb{V}[a, b](c f .(4.4))$, we see that (6.4)-(6.6), (3.6), (4.1) and (4.2) give

$$
\begin{aligned}
\left\|x_{i}\right\|_{\infty} \leq & 3\left(1+\frac{\|v\|_{\infty}}{|k|}\right)(b-a)\|h\|_{\infty}+\frac{\left|c_{0}\right|}{|k|} \\
& +\left(1+\frac{\|v\|_{\infty}}{|k|}\right) J_{0}, \quad i=1,2 .
\end{aligned}
$$

Due to (6.8)-(6.10), we see that $x_{i} \in \mathbb{W}^{1, \infty}[a, b], i=1,2$, and the operator $\mathcal{F}$ is defined well.

Lemma 6.1 Assume that (6.1) holds and that $\Omega$ and $\mathcal{F}$ are given by (6.2) and (6.5), (6.6), respectively. Then the operator $\mathcal{F}$ is compact on $\bar{\Omega}$.

\section{Proof}

Step 1 . We show that $\mathcal{F}$ is continuous on $\bar{\Omega}$. Choose a sequence

$$
\left\{u^{[n]}\right\}_{n=1}^{\infty}=\left\{\left(u_{1}^{[n]}, u_{2}^{[n]}\right)\right\}_{n=1}^{\infty} \subset \bar{\Omega}
$$

which is convergent in $\mathbb{W}^{1, \infty}[a, b] \times \mathbb{W}^{1, \infty}[a, b]$, that is, $(c f .(1.5))$ there exists $u=\left(u_{1}, u_{2}\right) \in \bar{\Omega}$ such that

$$
\lim _{n \rightarrow \infty}\left\|u_{1}^{[n]}-u_{1}\right\|_{1, \infty}=0, \quad \lim _{n \rightarrow \infty}\left\|u_{2}^{[n]}-u_{2}\right\|_{1, \infty}=0
$$

Lemma 5.1 and Lemma 5.2 yield

$$
\mathcal{P} u_{1}, \mathcal{P} u_{1}^{[n]} \in\left[a_{1}, b_{1}\right], \quad n \in \mathbb{N}, \quad \lim _{n \rightarrow \infty} \mathcal{P} u_{1}^{[n]}=\mathcal{P} u_{1},
$$

where $\mathcal{P}$ is defined by (5.4). Denote

$$
x=\left(x_{1}, x_{2}\right)=\mathcal{F}\left(u_{1}, u_{2}\right), \quad x^{[n]}=\left(x_{1}^{[n]}, x_{2}^{[n]}\right)=\mathcal{F}\left(u_{1}^{[n]}, u_{2}^{[n]}\right), \quad n \in \mathbb{N} .
$$

We will prove that

$$
\lim _{n \rightarrow \infty}\left\|x_{1}^{[n]}-x_{1}\right\|_{1, \infty}=0, \quad \lim _{n \rightarrow \infty}\left\|x_{2}^{[n]}-x_{2}\right\|_{1, \infty}=0
$$

By (4.7), (6.8), (6.11) and (6.13),

$$
\lim _{n \rightarrow \infty}\left\|\left(x_{i}^{[n]}\right)^{\prime}-x_{i}^{\prime}\right\|_{\infty}=\lim _{n \rightarrow \infty}\left\|f\left(\cdot, u_{i}^{[n]}(\cdot)\right)-f\left(\cdot, u_{i}(\cdot)\right)\right\|_{\infty}=0, \quad i=1,2 .
$$

Using (4.1), we get

$$
\lim _{n \rightarrow \infty}\left|\int_{\tau}^{\tau_{n}}\right| f\left(s, u_{1}^{[n]}(s)\right)-f\left(s, u_{2}^{[n]}(s)\right)|\mathrm{d} s| \leq 2 \lim _{n \rightarrow \infty}\left|\int_{\tau}^{\tau_{n}} h(s) \mathrm{d} s\right|=0 .
$$


Since

$$
\begin{aligned}
\int_{a}^{b}\left(f_{u^{[n]}}(s)-f_{u}(s)\right) \mathrm{d} s= & \int_{a}^{\tau}\left(f\left(s, u_{1}^{[n]}(s)\right)-f\left(s, u_{1}(s)\right)\right) \mathrm{d} s \\
& +\int_{\tau}^{b}\left(f\left(s, u_{2}^{[n]}(s)\right)-f\left(s, u_{2}(s)\right)\right) \mathrm{d} s \\
& +\int_{\tau}^{\tau_{n}}\left(f\left(s, u_{1}^{[n]}(s)\right)-f\left(s, u_{2}^{[n]}(s)\right)\right) \mathrm{d} s,
\end{aligned}
$$

the Lebesgue dominated convergence theorem and (6.16) give

$$
\lim _{n \rightarrow \infty} \int_{a}^{b}\left|f_{u^{[n]}}(s)-f_{u}(s)\right| \mathrm{d} s=0
$$

Using (6.13) and (6.5), we get

$$
\begin{aligned}
\left|x_{1}^{[n]}(a)-x_{1}(a)\right| \leq & \int_{a}^{b}|G(a, s)| \cdot\left|f_{u^{[n]}}(s)-f_{u}(s)\right| \mathrm{d} s \\
& +\left|\frac{v\left(\mathcal{P} u_{1}^{[n]}\right)}{k} \mathcal{J}\left(\mathcal{P} u_{1}^{[n]}, u_{1}^{[n]}\left(\mathcal{P} u_{1}^{[n]}\right)\right)-\frac{v\left(\mathcal{P} u_{1}\right)}{k} \mathcal{J}\left(\mathcal{P} u_{1}, u_{1}\left(\mathcal{P} u_{1}\right)\right)\right| .
\end{aligned}
$$

The continuity and boundedness of $\mathcal{P}, \mathcal{J}$ and $v$ (cf. Lemma 5.2, (2.4), (4.2), (4.4) and (6.12)) imply

$$
\begin{aligned}
& \lim _{n \rightarrow \infty} \mid\left|\frac{v\left(\mathcal{P} u_{1}^{[n]}\right)}{k} \mathcal{J}\left(\mathcal{P} u_{1}^{[n]}, u_{1}^{[n]}\left(\mathcal{P} u_{1}^{[n]}\right)\right)-\frac{v\left(\mathcal{P} u_{1}\right)}{k} \mathcal{J}\left(\mathcal{P} u_{1}, u_{1}\left(\mathcal{P} u_{1}\right)\right)\right| \\
& \leq \frac{\|v\|_{\infty}}{|k|} \lim _{n \rightarrow \infty}\left|\mathcal{J}\left(\mathcal{P} u_{1}^{[n]}, u_{1}^{[n]}\left(\mathcal{P} u_{1}^{[n]}\right)\right)-\mathcal{J}\left(\mathcal{P} u_{1}, u_{1}\left(\mathcal{P} u_{1}\right)\right)\right| \\
& \quad+\frac{J_{0}}{|k|} \lim _{n \rightarrow \infty}\left|v\left(\mathcal{P} u_{1}^{[n]}\right)-v\left(\mathcal{P} u_{1}\right)\right|=0,
\end{aligned}
$$

wherefrom, by the boundedness of $G$ and (6.17),

$$
\lim _{n \rightarrow \infty}\left|x_{1}^{[n]}(a)-x_{1}(a)\right|=0 .
$$

Using (6.13) and integrating (6.8), we get

$$
x_{1}(t)=x_{1}(a)+\int_{a}^{t} f\left(s, u_{1}(s)\right) \mathrm{d} s, \quad x_{1}^{[n]}(t)=x_{1}^{[n]}(a)+\int_{a}^{t} f\left(s, u_{1}^{[n]}(s)\right) \mathrm{d} s,
$$

and, due to (6.15) and (6.18), we arrive at

$$
\lim _{n \rightarrow \infty}\left\|x_{1}^{[n]}-x_{1}\right\|_{\infty}=0
$$

Similarly, we derive

$$
\lim _{n \rightarrow \infty}\left|x_{2}^{[n]}(b)-x_{2}(b)\right|=0, \quad \lim _{n \rightarrow \infty}\left\|x_{2}^{[n]}-x_{2}\right\|_{\infty}=0 .
$$

Properties (6.15), (6.19) and (6.20) yield (6.14). 
Step 2. We show that the set $\mathcal{F}(\bar{\Omega})$ is relatively compact in $\mathbb{W}^{1, \infty}[a, b] \times \mathbb{W}^{1, \infty}[a, b]$. Choose an arbitrary sequence

$$
\left\{\left(x_{1}^{[n]}, x_{2}^{[n]}\right)\right\}_{n=1}^{\infty} \subset \mathcal{F}(\bar{\Omega}) \subset \mathbb{W}^{1, \infty}[a, b] \times \mathbb{W}^{1, \infty}[a, b] .
$$

We need to prove that there exists a convergent subsequence. Clearly, there exists $\left\{\left(u_{1}^{[n]}, u_{2}^{[n]}\right)\right\}_{n=1}^{\infty} \subset \bar{\Omega}$ such that

$$
\mathcal{F}\left(u_{1}^{[n]}, u_{2}^{[n]}\right)=\left(x_{1}^{[n]}, x_{2}^{[n]}\right), \quad n \in \mathbb{N} .
$$

Choose $i \in\{1,2\}$. By (5.1) and (6.2), it holds

$$
\begin{aligned}
& \left\{u_{i}^{[n]}\right\}_{n=1}^{\infty} \subset \mathbb{W}^{1, \infty}[a, b], \quad\left\|u_{i}^{[n]}\right\|_{\infty} \leq K, \\
& \left|u_{i}^{[n]}\left(t_{1}\right)-u_{i}^{[n]}\left(t_{2}\right)\right|=\left|\int_{t_{1}}^{t_{2}}\left(u_{i}^{[n]}\right)^{\prime}(s) \mathrm{d} s\right| \leq\|h\|_{\infty}\left|t_{1}-t_{2}\right|
\end{aligned}
$$

for $t_{1}, t_{2} \in[a, b], n \in \mathbb{N}$. Therefore, the Arzelà-Ascoli theorem yields that there exists a subsequence

$$
\left\{\left(u_{1}^{[m]}, u_{2}^{[m]}\right)\right\}_{m=1}^{\infty} \subset\left\{\left(u_{1}^{[n]}, u_{2}^{[n]}\right)\right\}_{n=1}^{\infty}
$$

which converges in $\mathbb{C}[a, b] \times \mathbb{C}[a, b]$. Consequently, for each $\varepsilon>0$, there exists $m_{0} \in \mathbb{N}$ such that for each $m \in \mathbb{N}$,

$$
m \geq m_{0} \quad \Rightarrow \quad\left\|u_{i}^{\left[m_{0}\right]}-u_{i}^{[m]}\right\|_{\infty}<\varepsilon, \quad i=1,2 .
$$

Similarly as in Step 1, we prove (cf. (6.15), (6.19), (6.20))

$$
\left\|\left(x_{i}^{\left[m_{0}\right]}\right)^{\prime}-\left(x_{i}^{[m]}\right)^{\prime}\right\|_{\infty}<\varepsilon, \quad\left\|x_{i}^{\left[m_{0}\right]}-x_{i}^{[m]}\right\|_{\infty}<\varepsilon, \quad i=1,2,
$$

which gives by (1.5) that $\left\{\left(x_{1}^{[m]}, x_{2}^{[m]}\right)\right\}_{m=1}^{\infty}$ is convergent in $\mathbb{W}^{1, \infty}[a, b] \times \mathbb{W}^{1, \infty}[a, b]$.

Remark 6.2 If there exists $\tau_{0} \in\left[a_{1}, b_{1}\right]$ such that $\gamma(x)=\tau_{0}$ for $x \in[-K, K]$, then problem (2.1)-(2.3) has an impulse at fixed time $\tau_{0}$ and a standard operator $\mathcal{F}_{0}$, acting on the space of piece-wise continuous functions on $[a, b]$ and having the form

$$
\left(\mathcal{F}_{0} z\right)(t)=\int_{a}^{b} G(t, s) f(s, z(s)) \mathrm{d} s+\frac{c_{0}}{k}+G\left(t, \tau_{0}\right) \mathcal{J}\left(\tau_{0}, z\left(\tau_{0}\right)\right), \quad t \in[a, b],
$$

can be used instead of the operator $\mathcal{F}$ from (6.5), (6.6). But this is not possible if $\gamma$ is not constant on $[-K, K]$. The reason is that then an impulse is realized at a state-dependent point $\tau=\gamma(z(\tau))$, and $\mathcal{F}_{0}$ with $\tau$ instead of $\tau_{0}$ should be investigated on the space $\mathbb{G}_{L}[a, b]$. But if we write a state-dependent $\tau$ instead of a fixed $\tau_{0}$ in $(6.21), \mathcal{F}_{0}$ loses its continuity on $\mathbb{G}_{L}[a, b]$, which we show in the next example. 
Example 6.3 Let $a=0, b=2$ and $\ell$ be from (2.5) with $k \in \mathbb{R}, k \neq 0$ and $v \in \mathbb{C}[0,2]$. Consider the functions

$$
u(t)=1, \quad u_{n}(t)=1-\frac{1}{n}, \quad t \in[0,2], n \in \mathbb{N} .
$$

Clearly, $u_{n} \rightarrow u$ uniformly on $[0,2]$ and hence

$$
\lim _{n \rightarrow \infty}\left\|u_{n}-u\right\|_{\infty}=0
$$

For $n \in \mathbb{N}$, denote $x_{n}=\mathcal{F}_{0} u_{n}$ and $x=\mathcal{F}_{0} u$. Assume that the barrier $\gamma$ is given by the linear function $\gamma(x)=x$ on $\mathbb{R}$ and the impulse function $\mathcal{J}(t, x)=1$ for $t \in[0,2], x \in \mathbb{R}$. Then

$$
\begin{aligned}
& \tau=\gamma(u(\tau))=u(\tau)=1, \\
& \tau_{n}=\gamma\left(u_{n}\left(\tau_{n}\right)\right)=u_{n}\left(\tau_{n}\right)=1-\frac{1}{n}, \quad n \in \mathbb{N},
\end{aligned}
$$

and, according to (6.21), we have for $t \in[0,2]$

$$
\begin{aligned}
& x_{n}(t)=\int_{0}^{2} G(t, s) f\left(s, 1-\frac{1}{n}\right) \mathrm{d} s+\frac{c_{0}}{k}+G\left(t, 1-\frac{1}{n}\right), \quad n \in \mathbb{N}, \\
& x(t)=\int_{0}^{2} G(t, s) f(s, 1) \mathrm{d} s+\frac{c_{0}}{k}+G(t, 1) .
\end{aligned}
$$

Consequently,

$$
\begin{aligned}
\lim _{n \rightarrow \infty}\left(x_{n}(1)-x(1)\right)= & \lim _{n \rightarrow \infty} \int_{0}^{2} G(1, s)\left(f\left(s, 1-\frac{1}{n}\right)-f(s, 1)\right) \mathrm{d} s \\
& +\lim _{n \rightarrow \infty}\left(G\left(1,1-\frac{1}{n}\right)-G(1,1)\right) \\
= & 1-\frac{v(1)}{k}-\left(-\frac{v(1)}{k}\right)=1
\end{aligned}
$$

due to (3.6). Hence $x_{n}(1) \nrightarrow x(1)$ and we have also $\left\|x_{n}-x\right\|_{\infty} \nrightarrow 0$, and $\mathcal{F}_{0}$ is not continuous on $\mathbb{G}_{L}[0,2]$.

Lemma 6.1 results in the following theorem.

Theorem 6.4 Assume that (6.1) holds and that the set $\Omega$ is given by (6.2), where

$$
K \geq\left(1+\frac{\|v\|_{\infty}}{|k|}\right)\left(3(b-a)\|h\|_{\infty}+J_{0}\right)+\frac{\left|c_{0}\right|}{|k|}
$$

Further, let the operator $\mathcal{F}$ be given by (6.5), (6.6). Then $\mathcal{F}$ has a fixed point in $\bar{\Omega}$.

Proof By Lemma 6.1, $\mathcal{F}$ is compact on $\bar{\Omega}$. Due to (5.1), (6.2), (6.5), (6.6), (6.10) and (6.22),

$$
\mathcal{F}(\bar{\Omega}) \subset \bar{\Omega} .
$$

Therefore, the Schauder fixed point theorem yields a fixed point of $\mathcal{F}$ in $\bar{\Omega}$. 


\section{Main result}

The main result, which is contained in Theorem 7.1, guarantees the solvability of problem (2.1)-(2.3) provided the data functions $f, \mathcal{J}$ and $\gamma$ are bounded (cf. (4.1)-(4.3)). As it is mentioned in Remark 4.1, Theorem 7.1 serves as an existence principle which, in combination with the method of a priori estimates, can lead to more general existence results for unbounded $f$ and $\mathcal{J}$ and concrete boundary conditions.

Theorem 7.1 Assume that (6.1) and (6.22) hold. Then there exists a solution $z$ of problem (2.1)-(2.3) such that

$$
\|z\|_{\infty} \leq K .
$$

Proof By Theorem 6.4, there exists $u=\left(u_{1}, u_{2}\right) \in \bar{\Omega}$ which is a fixed point of the operator $\mathcal{F}$ defined in (6.5) and (6.6). This means that

$$
\begin{aligned}
& u_{1}(t)= \begin{cases}\int_{a}^{b} G(t, s) f_{u}(s) \mathrm{d} s+\frac{c_{0}}{k} & \\
\quad-\frac{v\left(\mathcal{P} u_{1}\right)}{k} \mathcal{J}\left(\mathcal{P} u_{1}, u_{1}\left(\mathcal{P} u_{1}\right)\right) & \text { if } t \leq \mathcal{P}_{u_{1}}, \\
\int_{a}^{b} G(t, s) f\left(s, u_{1}(s)\right) \mathrm{d} s+\frac{c_{0}}{k} & \\
\quad-\frac{v\left(\mathcal{P} u_{1}\right)}{k} \mathcal{J}\left(\mathcal{P} u_{1}, u_{1}\left(\mathcal{P} u_{1}\right)\right)+\mathcal{A}_{1} u & \text { if } t>\mathcal{P}_{u_{1}},\end{cases} \\
& u_{2}(t)=\left\{\begin{array}{rr}
\int_{a}^{b} G(t, s) f\left(s, u_{2}(s)\right) \mathrm{d} s+\frac{c_{0}}{k} & \\
\quad+\left(1-\frac{\nu\left(\mathcal{P} u_{1}\right)}{k}\right) \mathcal{J}\left(\mathcal{P} u_{1}, u_{1}\left(\mathcal{P} u_{1}\right)\right)+\mathcal{A}_{2} u & \text { if } t \leq \mathcal{P}_{u_{1}}, \\
\int_{a}^{b} G(t, s) f_{u}(s) \mathrm{d} s+\frac{c_{0}}{k} & \\
\quad+\left(1-\frac{\nu\left(\mathcal{P} u_{1}\right)}{k}\right) \mathcal{J}\left(\mathcal{P} u_{1}, u_{1}\left(\mathcal{P} u_{1}\right)\right) & \text { if } t>\mathcal{P}_{u_{1}},
\end{array}\right.
\end{aligned}
$$

where $G, \mathcal{P}, f_{u}, \mathcal{A}_{1}, \mathcal{A}_{2}$ are given by (3.6), (5.4), (6.3), (6.7), respectively. Recall that $\mathcal{P} u_{1}$ is a unique point in $(a, b)$ satisfying

$$
\mathcal{P} u_{1}=\tau_{1} \in\left[a_{1}, b_{1}\right], \quad \text { where } \tau_{1}=\gamma\left(u_{1}\left(\tau_{1}\right)\right) .
$$

For $t \in[a, b]$, define a function $z$ by

$$
z(t)= \begin{cases}u_{1}(t) & \text { if } t \in\left[a, \tau_{1}\right] \\ u_{2}(t) & \text { if } t \in\left(\tau_{1}, b\right]\end{cases}
$$

Differentiating (7.2), (7.3) and using (3.6) and (6.3), we get $u_{i}^{\prime}(t)=f\left(t, u_{i}(t)\right)$ for a.e. $t \in$ $[a, b], i=1,2$, and consequently

$$
z^{\prime}(t)=f(t, z(t)) \quad \text { for a.e. } t \in[a, b] .
$$

By virtue of (7.2)-(7.5), we have

$$
z\left(\tau_{1}+\right)-z\left(\tau_{1}\right)=u_{2}\left(\tau_{1}\right)-u_{1}\left(\tau_{1}\right)=\mathcal{J}\left(\tau_{1}, u_{1}\left(\tau_{1}\right)\right)=\mathcal{J}\left(\tau_{1}, z\left(\tau_{1}\right)\right) .
$$

Let us show that $\tau_{1}$ is a unique solution of the equation

$$
t=\gamma(z(t))
$$


in $[a, b]$. According to (7.4) and (7.5), it suffices to prove

$$
t \neq \gamma\left(u_{2}(t)\right), \quad t \in\left(\tau_{1}, b\right]
$$

Since $\left(u_{1}, u_{2}\right) \in \bar{\Omega}$, we have (cf. (5.1) and (6.2))

$$
\left\|u_{i}\right\|_{\infty} \leq K, \quad\left\|u_{i}^{\prime}\right\|_{\infty} \leq\|h\|_{\infty}, \quad i=1,2 .
$$

Assume that the first condition in (4.6) is fulfilled. Then $\mathcal{J}\left(\tau_{1}, x\right) \geq 0, \gamma^{\prime}(x) \leq 0$ for $x \in$ $[-K, K]$. Put

$$
\sigma(t)=\gamma\left(u_{2}(t)\right)-t, \quad t \in[a, b] .
$$

By (7.6), $u_{2}\left(\tau_{1}\right)-u_{1}\left(\tau_{1}\right)=\mathcal{J}\left(\tau_{1}, u_{1}\left(\tau_{1}\right)\right) \geq 0$, and since $\gamma$ is non-increasing, we have

$$
\sigma\left(\tau_{1}\right)=\gamma\left(u_{2}\left(\tau_{1}\right)\right)-\tau_{1} \leq \gamma\left(u_{1}\left(\tau_{1}\right)\right)-\tau_{1}=0
$$

due to (7.4). Using (4.5), we derive for $t \in\left(\tau_{1}, b\right]$

$$
\begin{aligned}
\sigma(t) & =\int_{\tau_{1}}^{t}\left(\gamma^{\prime}\left(u_{2}(s)\right) u_{2}^{\prime}(s)-1\right) \mathrm{d} s \leq \int_{\tau_{1}}^{t}\left(\left|\gamma^{\prime}\left(u_{2}(s)\right)\right| \cdot\left\|u_{2}^{\prime}\right\|_{\infty}-1\right) \mathrm{d} s \\
& <\int_{\tau_{1}}^{t}\left(\frac{1}{\|h\|_{\infty}}\|h\|_{\infty}-1\right) \mathrm{d} s=0 .
\end{aligned}
$$

So, (7.8) is valid. If the second condition in (4.6) is fulfilled, we use the dual arguments.

Finally, let us check that $\ell(z)=c_{0}$. By (7.2)-(7.6) and (3.6), we have

$$
z(t)=\int_{a}^{b} G(t, s) f(s, z(s)) \mathrm{d} s+\frac{c_{0}}{k}+G\left(t, \tau_{1}\right) \mathcal{J}\left(\tau_{1}, z\left(\tau_{1}\right)\right) .
$$

Put

$$
x(t)=\int_{a}^{b} G(t, s) f(s, z(s)) \mathrm{d} s .
$$

Then, according to (iii) of Definition 3.3 and Remark 3.2, we get $\ell(x)=0$. Further, using (3.7) from Lemma 3.5, we arrive at $\ell\left(G\left(\cdot, \tau_{1}\right)\right)=0$. Consequently, due to (2.5), (7.9) and (7.10), $\ell(z)$ results in

$$
\begin{aligned}
\ell(z) & =\ell(x)+\ell\left(\frac{c_{0}}{k}\right)+\ell\left(G\left(\cdot, \tau_{1}\right)\right) \mathcal{J}\left(\tau_{1}, z\left(\tau_{1}\right)\right) \\
& =\ell\left(\frac{c_{0}}{k}\right)=k \frac{c_{0}}{k}+{ }_{(\mathrm{KS})} \int_{a}^{b} v(t) \mathrm{d}\left[\frac{c_{0}}{k}\right]=c_{0} .
\end{aligned}
$$

The authors declare that they have no competing interests. 


\section{Acknowledgements}

This research was supported by the grant Matematické modely, PrF_2013_013. The authors thank the referees for suggestions which improved the paper.

Received: 13 May 2013 Accepted: 13 August 2013 Published: 28 August 2013

\section{References}

1. Bainov, D, Simeonov, P: Impulsive Differential Equations: Periodic Solutions and Applications. Longman, Harlow (1993)

2. Lakshmikantham, V, Bainov, DD, Simeonov, PS: Theory of Impulsive Differential Equations. World Scientific, Singapore (1989)

3. Samoilenko, AM, Perestyuk, NA: Impulsive Differential Equations. World Scientific, Singapore (1995)

4. Bajo, I, Liz, E: Periodic boundary value problem for first order differential equations with impulses at variable times. J. Math. Anal. Appl. 204, 65-73 (1996)

5. Belley, J, Virgilio, M: Periodic Duffing delay equations with state dependent impulses. J. Math. Anal. Appl. 306, 646-662 (2005)

6. Belley, J, Virgilio, M: Periodic Liénard-type delay equations with state-dependent impulses. Nonlinear Anal. TMA 64, 568-589 (2006)

7. Frigon, M, O'Regan, D: First order impulsive initial and periodic problems with variable moments. J. Math. Anal. Appl. 233, 730-739 (1999)

8. Benchohra, M, Graef, JR, Ntouyas, SK, Ouahab, A: Upper and lower solutions method for impulsive differential inclusions with nonlinear boundary conditions and variable times. Dyn. Contin. Discrete Impuls. Syst., Ser. A, Math. Anal. 12, 383-396 (2005)

9. Frigon, M, O'Regan, D: Second order Sturm-Liouville BVP's with impulses at variable times. Dyn. Contin. Discrete Impuls. Syst., Ser. A, Math. Anal. 8, 149-159 (2001)

10. Rachůnková, I, Tomeček, J: A new approach to BVPs with state-dependent impulses. Bound. Value Probl. 2013, 22 (2013)

11. Rachůnková, I, Tomeček, J: Second order BVPs with state-dependent impulses via lower and upper functions. Cent. Eur. J. Math. (to appear)

12. Rachůnková, I, Tomeček, J: Existence principle for BVPs with state-dependent impulses. Topol. Methods Nonlinear Anal. (submitted)

13. Tvrdý, M: Linear integral equations in the space of regulated functions. Math. Bohem. 123, 177-212 (1998)

14. Tvrdý, M: Regulated functions and the Perron-Stieltjes integral. Cas. Pěst. Mat. 114, 187-209 (1989)

15. Schwabik, Š, Tvrdý, M, Vejvoda, O: Differential and Integral Equations. Academia, Prague (1979)

16. Rachůnková, I, Tomeček, J: Singular Dirichlet problem for ordinary differential equation with impulses. Nonlinear Anal. TMA 65, 210-229 (2006)

17. Rachůnková, I, Tomeček, J: Impulsive BVPs with nonlinear boundary conditions for the second order differential equations without growth restrictions. J. Math. Anal. Appl. 292, 525-539 (2004)

doi:10.1186/1687-2770-2013-195

Cite this article as: Rachůnek and Rachůnková: First-order nonlinear differential equations with state-dependent impulses. Boundary Value Problems 2013 2013:195.

\section{Submit your manuscript to a SpringerOpen ${ }^{\circ}$ journal and benefit from:}

- Convenient online submission

Rigorous peer review

- Immediate publication on acceptance

- Open access: articles freely available online

- High visibility within the field

- Retaining the copyright to your article 